\title{
High Speed Cinemicrographic Studies on Rabbit Tracheal (Ciliated) Epithelia: Determination of the Beat Pattern of Tracheal Cilia
}

\author{
ANTHONY T. W. CHEUNG \\ California Institute of Technology, Pasadena, California, USA \\ THEODORE L. JAHN \\ University of California, Los Angeles, California, USA
}

\section{Extract}

High speed cinemicrographs reveal that the ciliary configuration and beat pattern of rabbit tracheal cilia differ significantly from classic descriptions, although the basic forward and return pattern is still observed. The tracheal cilia are short and stout (about $6 \mu \mathrm{m}$ in length) and are of a slightly bent original configuration. At the start of the forward stroke, the cilia bear about $75-90^{\circ}$ to the epithelial surface in the direction of the forward stroke. The forward stroke is planar and consists of a simple "bowing" movement, without showing any progressive bending and without changing the original ciliary configuration. The complete forward stroke sweeps through an arc of about $35-40^{\circ}$, with the tips of the cilia penetrating the bottom of the mucous layer through an arc of about $5-8^{\circ}$ at the start of each forward stroke. The return stroke starts from close to the epithelial surface and returns to the starting position of the forward stroke by reversing the planer forward pathway at a reduced speed. The stout nature of the cilia, the original bent ciliary configuration, the near vertical starting position of the forward stroke, the extent of the angular sweeps of the strokes, the peculiar "bowing" forward stroke movement, and the nature of the return stroke all serve to enhance efficiency in moving fluid forward.

It has been documented that each forward stroke of the tracheal cilia causes and maintains a swift forward movement of the intermediate serous (liquid) layer of the mucociliary system. This movement, in turn, reacts hydrodynamically with the top mucous layer, and together with the clawing action of the tips of the cilia at the start of the forward stroke, causes the mucous layer to move cephalad in the manner of a conveyor belt.

\section{Speculation}

This research report describes a method by which the ciliary activity of live rabbit tracheal cilia is documented. The exact ciliary beat pattern can then be determined and the clearance mechanism of tracheal mucus better understood.

It is speculated that tracheal mucus stagnation in cystic fibrosis may be a hydromechanical and biochemical problem rather than a direct cilia control problem. Detailed analysis of the ciliary beat pattern and mucus clearance of the tracheal mucociliary system may serve to provide a reference baseline to investigate the possible physiologic effects of cystic fibrosis serum on ciliated epithelial cells (such physiologic effects will be dealt with in a separate report).

The mucociliary system of mammalian trachea is made up of three components $(7,8,10,11)$. Physiologists have long realized the significance of this layered component system as a clearance mechanism for foreign particles in mammalian trachea, although the exact detailed mechanism was still unknown. It was a generally accepted concept that the top mucous layer was moved cephalad by the tracheal cilia. How this mucus transport was brought about and the reason why the return stroke did not drag the mucous layer back were the two key questions that scientists had failed to solve. What made the situation more complicated was that the exact basic beat pattern of the tracheal cilia was also unk nown.

Up until now, not much work has been conducted on the ciliary activities of mammalian tracheal epithelia. Compared with the vast volume of publications on the ciliary beat pattern of various ciliates, the description on the beat pattern of tracheal cilia is extremely inadequate. There are many reasons for this: that such cilia are not readily accessible for study since they occur in tracts in which individual cilium cannot be seen or reached; that the ciliary beat pattern itself appears to be so variable and complicated to the human eye; furthermore, that such cilia cannot be directly observed and analyzed under the microscope because of their small size, their high angular velocity, their frequency of beat, their density of distribution, and their unique optical properties which are almost identical with those of the underlying epithelial cells.

This report describes a method to document the ciliary activities of live rabbit tracheal cilia by high speed cinemicrography. Based on such documented data, the detailed ciliary beat pattern and the tracheal mucus clearance mechanism of the tracheal mucociliary system can then be determined.

\section{MATERIALS AND METHODS}

\section{BIOLOGIC MATERIAL}

Ciliated epithelia of rabbit trachea were obtained from New Zealand White rabbits (ranging from $12-15 \mathrm{lbs}$.) purchased from biologic supply firms in Southern California. The rabbits were anesthetized by intravenous injections of sodium pentobarbital (Diabutal) at a dosage dependent on body weights. The epithelia were microdissected out from the trachea and were immediately suspended in culture medium 199, enriched with glutamine and Hank's salt. Active and Healthy epithelia were selected and used immediately to document the form of ciliary beat by high speed cinemicrography (in Nomarski optics), although they could be kept alive for over a week in culture chambers.

\section{CHEMICAL MATERIAL}

Culture medium 199 was obtained from Grand Island Biological Company in Berkely, Calif. The medium was stored at $4^{\circ}$ and buffered at $\mathrm{pH}$ 7.4. This culture medium was enriched with glutamine and Hank's salt.

\section{OPTICAL EQUIPMENT}

Camera. Two 16-mm high speed cameras were used: a Redlake Locam high speed camera, model 162-4DC, and a Milliken 
Teledyne high speed camera, model DBM-55. Both cameras were of pin registry and could manage an operating speed of up to 500 frames/sec.

Microscope. The movie camera was attached to a Zeiss research microscope (either a Zeiss Universal or a Zeiss Standard) equipped with Nomarski differential interference contrast, phase contrast, and dark field optics. The light path of the optics had to be very carefully aligned to give the best results. This high speed cinemicrographic set-up was arranged as shown in Figure 1.

Light Source. Two different light sources were used: a tungstenhalogen 100-W source (at UCLA) and a Chadwick-Helmuth camera-modulated xenon strobe (at Caltech).

Timing Light. The exact framing rate of the high speed cinemicrographs (movies) could be determined by an internal timing light which was projected onto the edge of the film as a reference record.

Film. Eastman Kodak Ektachrome commercial 7225 ECO films were used for color work, while Eastman Kodak 4X negative 4XN and Kodak RAR stock 2475 recording films were used for black and white work. Framing rates of up to $500 \mathrm{frames} / \mathrm{sec}$ could be used for these types of fast films in black and white documentations.

Movie Analyzer. Analysis of the high speed cinemicrographs were made by repeated viewing at various speeds (2-24 frames/ sec) with a flicker-less L.W. photo-optical analyzer, and also by utilization of a frame-by-frame tracing analytic procedure with the aid of a Traid R-100 film reader. Black and white photographic prints were made from selected movie sequences for analytical purposes.

\section{METHODS}

Microdissected tracheal epithelia were suspended in enriched culture medium 199. Segments with active ciliary movement and smooth cell margins were selected and placed under a Vaseline mount to be photographed with the high speed cine-

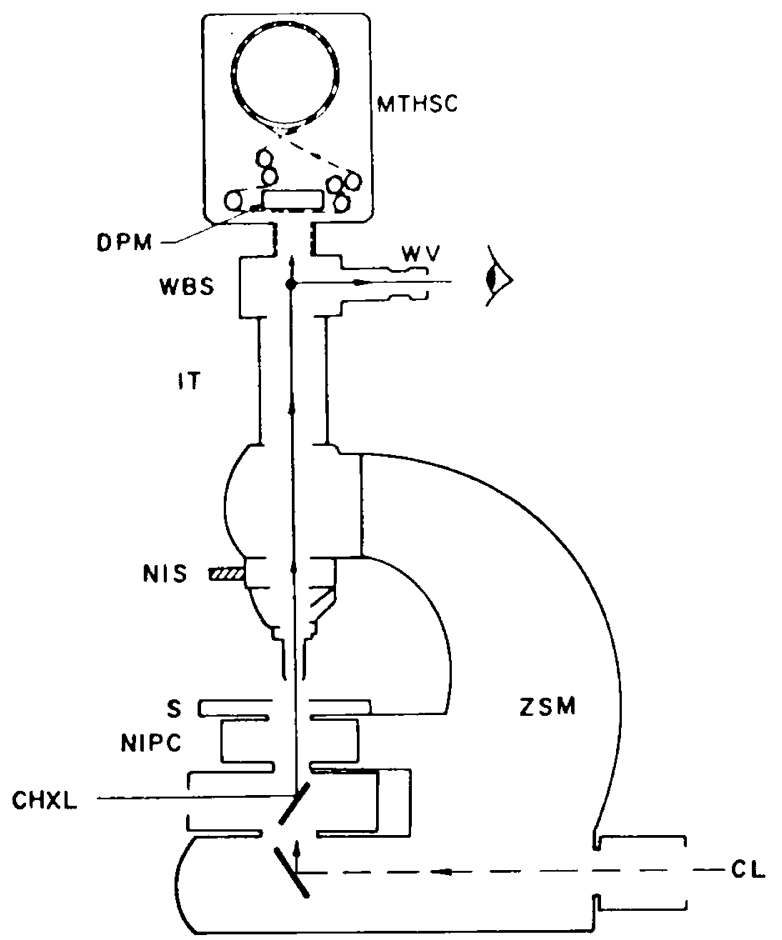

Fig. 1. Optical arrangement for high speed cinemicrography. The simplified light path is indicated by lines with arrows. MTHSC: Milliken Teledyne high speed camera; $D P M$ : pin registry mechanism; $W V$ : Wild viewer; WBS: Wild beam splitter; IT: intermediate tube; $N I S$ : Nomarski interference-phase (achromatic-aplanatic) 1.4 N.A. condenser; ZSM: Zeiss standard research microscope; $C H X L$ : Chadwick-Helmuth xenon strobe light source; $C L$ : standard Zeiss tungsten light source. micrographic set-up under Nomarski optics. The films were developed and processed in our own laboratory. Acufine was used in the developing process to yield a high ASA of over 3,000. Subsequent analysis of the high speed cinemicrographs served to illustrate the detailed form of ciliary activities and mucus transport.

Two separate categories of high speed cinemicrographs were taken: (l) ciliary activities of the tracheal epithelia with the mucous and the intermediate serous (liquid) layers intact; (2) ciliary activities of the tracheal epithelia with the mucous and the intermediate serous (liquid) layers removed, and with the cilia beating actively in the enriched culture medium.

Fluid tracers (DC polystyrene latex spheres) of diameters 0.5 $1.0 \mu \mathrm{m}$ were occasionally introduced into the medium to illustrate the velocity and the movement patterns of the fluid bathing the cilia (as well as the transport of mucus) with reference to the forward and return strokes.

Surface view cinemicrographs as well as profile (side) view cinemicrographs were taken. The surface view cinemicrographs were taken to confirm the planar nature of the ciliary beat pattern and the movement of the mucous layer. The profile view cinemicrographs were focused to show the relationship of the cilia with reference to the various layers of the mucociliary system, the extent and degree of penetration of the tips of the cilia to the mucous layer during the forward stroke and the angular sweeps of the forward and return strokes. Such photographic achievements were made possible through the utilization of the unique "optical section" focusing characteristics (12) of the Nomarski optics.

Observations and analyses were also made on the open trachea of anesthetized rabbits (open window technique) to study the in vivo flow rate of the mucous layer and the transport of foreign particles on top of it.

\section{OBSERVATIONS AND RESULTS}

The form of beat of tracheal cilia is determined from slow motion analysis $(2-24$ frames/sec) or frame-by-frame tracing of the cinemicrographs taken with Nomarski optics. The velocity and pathways of the fluid tracers (as obtained from analysis of the cinemicrographs) have been recorded to interpret the velocity of the cilia and the local flow velocity of the various mucociliary layers with reference to the frequencies of ciliary beat and the various stages of the forward and return strokes.

The average length of rabbit tracheal cilia is $6.1 \pm 0.5 \mu \mathrm{m}$. The frequency of beat of the tracheal cilia is $22 \pm 2 \mathrm{~Hz}$ (at $21^{\circ}$ ) with the mucous layer intact, and more variable (about $20-30 \mathrm{~Hz}$ ) with the mucous layer removed and the cilia beating in culture medium 199 at $21^{\circ}$. The forward-return velocity ratio is $3.4 \pm 0.3$, with the maximum velocity occurring at the tips of the cilia. The average rate of mucus transport is about $250 \pm 50 \mu \mathrm{m} / \mathrm{sec}\left(\right.$ at $21^{\circ}$ ) with the tip velocity of the cilia reaching a peak of $600 \mu \mathrm{m} / \mathrm{sec}$.

Cinemicrographs (at a magnification of 795 times) reveal that the tracheal cilia are very densely packed (average density of distribution is $5.0 \pm 0.5 \mathrm{cilia} / \mu \mathrm{m}^{2}$ ). However, this density distribution is very misleading as not all the tracheal epithelial cells are ciliated.

The tracheal cilia beat in good synchrony with cilia from the same epithelial cell and most of the time out of phase with those of neighboring cells. A loose pattern of beat coordination is apparent, but not synchronized enough to show any definite metachrony.

The origi: al postural configuration of the tracheal cilia at their starting position is slightly bent (as illustrated in Figs. 2, 3, and 4) and the forward stroke normally starts at a position bearing about $85^{\circ}$ to the epithelial surface in the direction of the forward stroke. The starting position varies with experimental conditions, ranging from $75^{\circ}$ to $95^{\circ}$; normally around $85^{\circ}$ and seldom over $90^{\circ}$. The return stroke which is slower than the forward stroke and returns from close to the epithelial surface (at about $35-40^{\circ}$ ) to the starting position. Figure $3, A$ and $B$, shows diagrammatically two tracheal cilia through one complete cycle of their beat with the configuration variations and angular sweeps outlined clearly. 
The forward stroke consists of a simple "bowing" movement sweeping through a planar arc of about $40^{\circ}$. The return stroke simply reverses the pathway of the forward stroke at a slower speed. The simple "bowing" movement pattern of the tracheal cilia and their return stroke differ remarkably from the classic progressive bending pattern for ciliary movement $(9,11)$ and also differ remarkably from the generally accepted ciliary beat pattern as postulated for tracheal cilia (11). The starting position of the forward stroke and the extent of the forward sweep in the beat process also differ remarkably from previous concepts.

The rate of mucus transport at various tracheal ciliary beat frequencies are obtained from the open window technique on anesthetized rabbits. This data has been interpreted with the cilia tip velocity of the various stages of the beat pattern and can serve to explain the biomechanics of tracheal mucus clearance. Such data will be presented and interpreted in detail in a separate report (5).

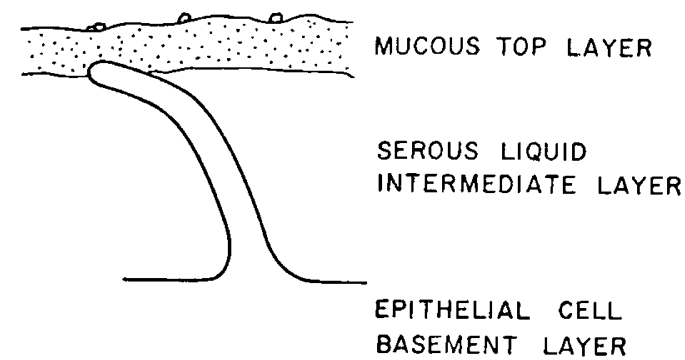

Fig. 2. A diagrammatic representation of the rabbit tracheal mucociliary system. The cilium is slightly bent and the starting position of the forward stroke bears about $75-90^{\circ}$ to the epithelial surface in the direction of the forward stroke. The tip of the cilium is in contact with the bottom of the mucous layer at the start of the forward stroke. Contact will be maintained through an arc of $5.8^{\circ}$.

\section{DISCUSSION}

It is generally belived that there is a mucous blanket enveloping the cilia of the tracheal epithelial cells, but the exact nature of the tracheal mucociliary system is not normally described in detail ( 1 , $2,6)$. However, the layered structure of this tracheal mucociliary system is actually more complicated than just a mucus blanket, as this system had been described as consisting of three components $(7,8,10,11)$. The bottom layer of epithelial cells (with the cilia) is the basement component with the cilia beating in an intermediate serous (liquid) layer. On top of this intermediate layer is the top mucous layer, the unidirectional flow of which will serve to protect the underlying cells against the adverse effects of inhaled material by the removal of foreign particles and liquid droplets out of the system.

In order to account for the biomechanics of mammalian tracheal mucus clearance, the first problem that has to be solved is the determination of the actual beat pattern of tracheal cilia and the movement of the mucus with reference to the forward and return strokes.

It is revealed in the high speed cinemicrographs taken with Nomarski optics that the cilia of rabbit trachea beat in a forward-and-return planar manner that differs significantly from classic concepts $(9,11)$. Previous descriptions of the tracheal ciliary beat pattern (11), although similar in nature in that the strokes are planar and in the classic forward-and-return pattern, differ significantly in the ciliary configuration, the actual beat pattern and the extent of the stroke sweeps. Furthermore, previous descriptions cannot account for the efficiency in moving the surrounding liquid and the apparent absence of dragging back of the liquid as well as the mucous layer.

Figures 2 and 3 show diagrammatically the position of the tip of a typical cilium with reference to the mucous layer during part of its forward stroke. The tip of the cilium penetrates the bottom of the mucous layer at the start of the forward stroke and this contact (a)

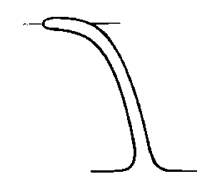

FORWARD STROKE (a)

(b)

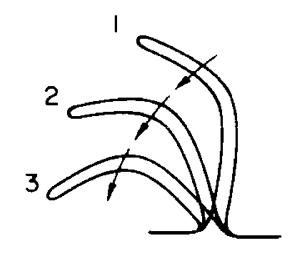

(c)

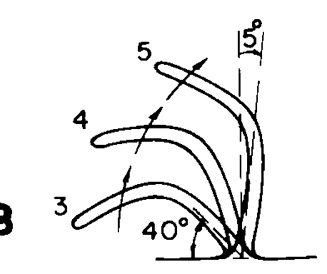

(c)

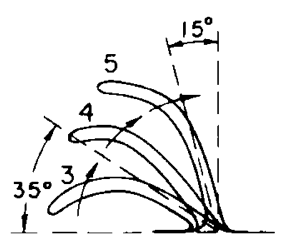

FORWARD STROKE

(b)
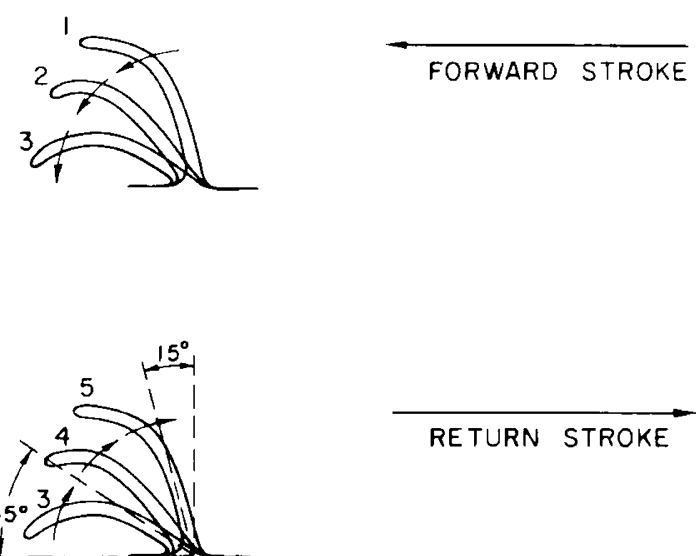

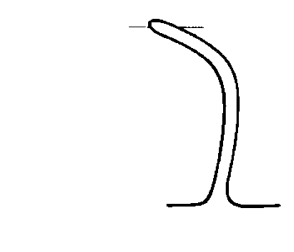
.

.




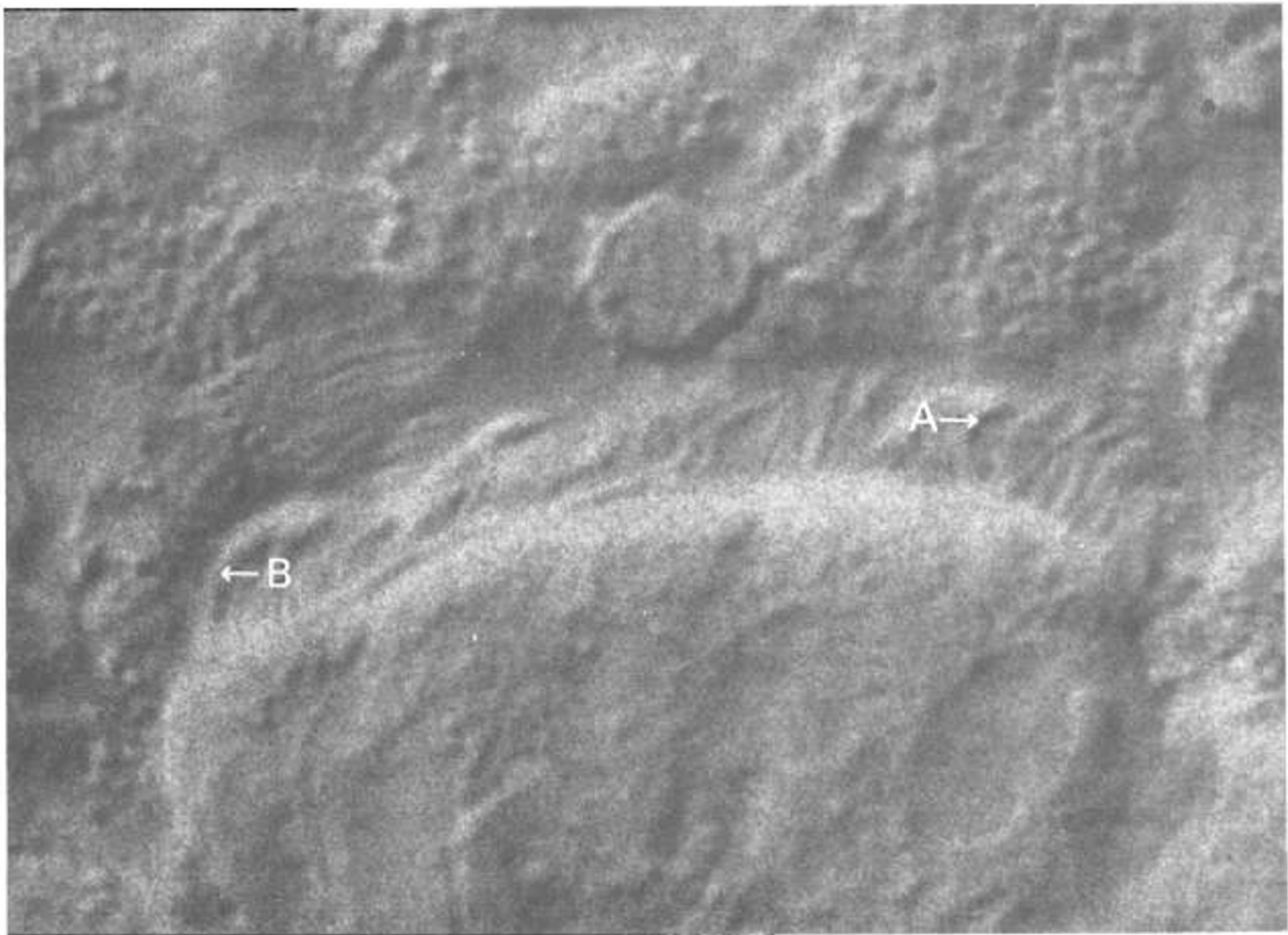

Fig. 4. A print of segment of rabbit tracheal (ciliated) epithelium from a 16-mm high speed movie sequence. Marker $A(\rightarrow)$ points to a cilium at the start of the forward stroke and marker $B(\leftarrow)$ points to a cilium that is about half way through its forward stroke. Note the characteristic "bent" original posture of the tracheal cilia. This piece of epithelium consists of five epithelial cells. (Magnification, $\times$ 645.)

is maintained through an arc of about $58^{\circ}$ only. Such a penetration contact is not present in the return stroke. This brief penetration contact at the start of the forward stroke is not continuous enough to explain the overall mucus transport phenomenon, but it definitely takes an active part in it (5). The absence of the penetration contact in the return stroke also helps to minimize the actual physical dragging back of the mucous layer.

Technically, it is virtually impossible to take pictures of the cilia tip penetration of the mucus with normal bright field and even phase contrast optics. However, this problem is overcome with the introduction of the Nomarski differential interference contrast optics. Because of its unique optical design, the numerical aperture of the condenser can be fully utilized, making it possible to reach the limit of resolution, while limiting depth of field to a very narrow range. As a result, "optical sections" can be placed through the specimen by focusing on certain planes without interference by structures above or below (12). This particular optical advantage makes the Nomarski optics an indispensable tool to study tracheal ciliary beat pattern and mucus clearance.

Detailed analyses of the available high speed cinemicrographs reveal that the tracheal cilia, other than "clawing" the mucous layer to move at the start of the forward stroke (during the first $8^{\circ}$ of the $40^{\circ}$ forward sweep), also manage to "scoop" up a column of intermediate serous liquid and propel it forward with each forward stroke. The swift movement of the intermediate serous (liquid) layer with the forward strokes interacts hydrodynamically with the viscoelastic mucous layer on top of it. This frictional intermolecular interaction, together with the clawing action of the cilia tips, serves to cause the top mucous layer to move along with the intermediate serous (liquid) layer in the manner of a "conveyor belt," the detail mechanism of which is dealt with in separate reports
$(3,4,5)$. The stout nature of the cilia, the original bent ciliary configuration, the cilia-mucus penetration contact at the start of the forward stroke, the unconventional "bowing" forward stroke movement, and the near vertical $\left(7590^{\circ}\right)$ forward stroke starting position all serve to enhance the efficiency in moving the intermediate and mucous layers forward. The return stroke, which is much slower and returns from close to the epithelial surface, and with the absence of a cilia-mucus penetration contact, serves to minimize the dragging back of the mucociliary layers: a phenomenon that is expected hydrodynamically and confirmed by fluid tracer analysis.

\section{SUMMARY}

The high speed cinemicrographic documentation of the beat pattern of tracheal cilia (as described and interpreted in this report) reveals the existence of a pattern of ciliary movement that is extremely efficient in moving liquid undirectionally. A "conveyor belt" clearance mechanism has been proposed by Cheung (3). making use of this ciliary beat pattern as a key factor to account for the biomechanics of tracheal mucus clearance. Such a concept, together with quantitative data obtained in the analysis of the high speed cinemicrographs, is dealt with hydrodinamically in a separate report (5). Further research is now being conducted in the Biophysical Hydromechanics Laboratory at the California Institute of Technology to look further into this cilia-related mucus transport and tracheal clearance phenomenon.

\section{REFERENCES AND NOTHS}

1. Barnett, B., and Miller, C. E.: In: S. Jakowska: Interdisciplinart investigation of mucus product and transport. Ann. N.Y. Aciad. Sci., 130(3) (1966). 
2. Carson, S., Goldhamer, R., and Weinberg, M. S.: In: S. Jakowska: Interdisciplinary investigation of mucus product and transport. Ann. N.Y. Acad. Sci., /30(3) (1966).

3. Cheung, A. T. W.: Doctoral Thesis, 1973. University of California at Los Angeles.

4. Cheung, A. T. W., and Jahn, T. L.: Determination of the movement pattern of the epithelial cilia of rabbit trachea and the clearance mechanism of the tracheal muco-ciliary system. In: Proceedings of Symposium on Swimming and Flying in Nature, 1974 (California Institute of Technology, Pasadena, Calif., in press).

5. Cheung. A. T. W., and Wu, T. Y.: High speed cinemicrographic studies on rabbit tracheal (ciliated) epithelia: Biomechanics of tracheal mucus clearance (In preparation).

6. Falk, H. L., Kotin, P., and Rowlette, W.: In: Jakowska: Mucous secretion. Ann. N.Y. Acad. Sci. $106(2)$ (1963).

7. Fawcett, D. W., and Porter, K. R.: A study of the fine structure of ciliated epithelia. J. Morphol., 94:21 (1954).

8. Finerty, J. C., and Cowdry, E. V.: A Textbook of Histology, Ed. 5 (Lea \& Febiger, Philadelphia, 1960).

9. Gray, J.: Ciliary Movement (Cambridge University Press, Cambridge, England, 1928)

Copyright $\odot 1976$ International Pediatric Research Foundation, Inc.
10. Greep, R. O. (Editor): Histology, Ed. 2 (McGraw-Hill Book Co., New York 1966).

11. Lucas, A. M., and Douglas, L. C.: Principles underlying ciliary activity in the respiratory tract. Arch. Otolaryngol., 20: 518 (1934).

12. Zeiss Publications: Phase Contrast and Interference Contrast (Carl Zeiss Publications, West Germany).

13. We would like to express our deep gratitude to Professor Theodore $\mathrm{Y}$. Wu, Professor Stuart R. Keller, and Dr. Christopher Brennen for their extremely valuable discussions in biophysical fluid mechanics. This work was jointly sponsored by the Office of Naval Research. National Science Foundation, and the United States Public Health Service. Their continous support is gratefully acknowledged.

14. This work is sponsored primarily by National Science Foundation Grant No. GK $31161 X$ and Office of Naval Research Contract No. N00014-67-A-0094. 0012 at the California Institute of Technology, and in part by National Science Foundation Grant No. GB 24840 and United States Public Health Service Grant No. AM 14388 at the University of California at Los Angeles.

15. Requests for reprints should be addressed to: A. T. W. Cheung, Ph.D., Thomas Laboratory (Mail Code: 104-44), California Institute of Technology. Pasadena, Calif. 91125 (USA).

16. Accepted for publication October 14, 1975.

Printed in U.S.A.

Pediat. Res. 10: $144-147$ (1976)

Cinemicrography epithelial cystic fibrosis tracheal cilia

\title{
High Speed Cinemicrographic Studies on Rabbit Tracheal (Ciliated) Epithelia: Cytolytic Effect of Cystic Fibrosis Serum on Tracheal Epithelial Cells
}

\author{
ANTHONY T. W. CHEUNG \\ California Institute of Technology, Pasadena, California, USA
}

THEODORE L. JAHN

University of California, Los Angeles, California, USA

\section{Extract}

High speed cinemicrographs are made on the ciliary activity of rabbit tracheal cilia with the aid of Nomarski optics. The detailed nature of the ciliary beat pattern is determined from slow motion analysis of the high speed cinemicrographs (1-3). Such documented forms of ciliary beat pattern and the physiologic state of the tracheal epithelia are utilized as basic reference controls to investigate the physiologic effects of cystic fibrosis serum on ciliated epithelia and the cystic fibrotic tracheal mucus stagnation phenomenon.

Careful analyses of the high speed cinemicrographs reveal that cystic fibrosis serum has no effect on the rhythm and beat pattern of rabbit tracheal cilia. However, it is shown in controlled procedures that cystic fibrosis serum has a cytolytic effect on the tracheal epithelial cells and also at the cell junctions. Ciliary dyskinesis, as described in previous reports $(6-9)$, is actually a secondary effect of cytolysis and cell destruction.

\section{Speculation}

Tracheal mucus stagnation in cystic fibrosis is not a ciliary dyskinetic problem, but rather a hydrodynamic and biochemical one.

Cystic fibrosis is a pediatric disorder which exhibits a very broad and varied spectrum of symptoms. One of the most notable is the stagnation of tracheal mucus transport. Some research reports had described the apparent presence of a cystic fibrosis factor in the serum of cystic fibrotic patients; such a factor was assumed to be the cause of ciliary dyskinesis and asynchrony in the beat pattern of tracheal cilia which could subsequently cause a stagnation of tracheal mucus transport (6.9). However, such a factor had not been satisfactorily defined and the apparent dyskinetic effect on ciliary activity had never been repeated outside of these laboratories. What made the situation more complicated was that the actual detailed beat pattern of tracheal cilia was still unknown and all the above reports were based solely on visual observations without any reference control.

This report follows up on the high speed cinemicrographic technique which has been utilized in the documentation and determination of the beat pattern of rabbit tracheal cilia $\left(\begin{array}{ll}1 & 3\end{array}\right)$ and describes a method which serves to investigate the possible physiologic effects of cystic fibrosis serum on tracheal epithelial cells.

\section{MATERIALS AND METHODS}

\section{BIOLOGICAL MATERIAL}

Rabbit tracheal epithelia were obtained from New Zealand White rabbits purchased from biologic supply firms in Southern California. The rabbits were anesthetized by intravenous injections of sodium pentobarbital (Diabutal) in a dosage dependent on body 